\title{
LE REGARD DE DIB ET CAMUS SUR LE PAYSAGE ALGÉRIEN
}

\author{
BrigitTe Leguen \\ $U N E D$
}

\section{Resumen}

Mohamed Dib y Albert Camus ambos nacieron en Argelia rodeados por el mismo paisaje. En este artículo analizaremos cómo cada uno percibe el espacio y sus significados.

Palabras clave: literatura, espacio, paisaje.

\section{Résumé}

Mohammed Dib et Albert Camus sont nés tous deux en Algérie entourés du même paysage. Dans cet article nous analyserons comment chacun perçoit l'espace et ses significations.

Mots clés : littérature, espace, paysage.

Beaucoup de choses nous séparent; d'autres nous unissent. Le paysage est un de ces éléments qui donne aux hommes et surtout aux artistes, l'occasion du partage par la contemplation de certains espaces privilégiés avec lesquels le dialogue est possible.

Cette contemplation met en œuvre des attitudes et des aptitudes basées sur une expérience visuelle, cognitive, littéraire, poétique qui sera l'occasion d'explorer et de comprendre qui l'on est, d'où l'on vient. Car le paysage a beaucoup à voir aussi avec la mémoire, avec la nostalgie. Il est enfin le lieu de l'identité. L'identité réelle, originelle, l'identité fabriquée.

L'œuvre de Mohammed Dib cherche à nommer la relation entre le paysage et son peuple, entre le paysage et lui-même.

"Au commencement est le paysage » Dira Dib paraphrasant la Genèse.

Dans sa poésie, dans ses romans, dans ses commentaires autour des photos qu'il a prises dans les années 40 à Tlemcen, le paysage se définit comme un phénomène de l'existence, une expérience fondatrice qui va de l'extérieur à l'intérieur, de la superficie aux origines, à partir de cette ville de Tlemcen, Le Pays des sources, le cadre de son enfance, là où il 
a vécu avec sa famille, ses frères et sœurs. Les photographies prises en I946 et commentées en I994 dans le livre Tlemcen ou les lieux de l'écriture et dans L'Arbre à dires de I998 s'échafaudent sur un double sentiment d'appartenance, algérienne par la culture et française par la colonisation de la langue.

L'écart temporel entre la prise d'image photographique et le moment des commentaires est un entretemps bouleversé par l'Histoire de l'Algérie, la guerre, l'indépendance, l'expulsion de Mohammed Dib, son établissement en France pour une situation d'exil permanente.

Plus rien n'est semblable désormais, ni pour lui ni pour tous les algériens.

Pourtant le travail de déconstruction des stéréotypes qu'entreprend Dib est bien antérieur à la décolonisation. Ses premiers textes, dès les années 50, La Grande Maison, L'incendie, Le Métier à tisser répondent au besoin de donner à son pays, à son histoire, une forme littéraire qui propose sa vision de l'Algérie de l'intérieur à partir de son expérience personnelle, qui représente le monde arabe et kabyle où il est né en contraste avec celle des écrivains algériens français comme Emmanuel Roblés, Albert Camus ou Jules Roy plus marqués par la perspective eurocentrée.

Dans de nombreuses interviews, Dib prend ses distances avec les préjugés coloniaux tout en essayant d'établir le contact avec l'Autre, attentif à la vérité schizophrène qui se dédouble et varie selon que l'on soit dominé ou dominant.

L'acte d'écrire lui permet de rejeter l'image déformée du colonisé et de donner à voir ce qui l'amène à la révolte.

Dans ce contexte difficile, où les expériences s'échelonnent selon la place qu'on occupe, selon les expériences qu'on endure, selon les pré/jugés systématiques, la relation d'Albert camus à ce même espace s'avère fragile et parfois ambigüe.

Camus dans son discours de Suède revendique son appartenance à une génération d'écrivains d'Algérie. Au moment de sa mort prématurée, Dib coordonne un hommage à son ami «Camus l'Algérien » et beaucoup d'aspects de leur enfance et de leur jeunesse les rapprochent, même si d'autres les séparent indéfectiblement : Une enfance pauvre, un père 
absent, l'engagement politique et les premiers combats, la littérature, et la contemplation amoureuse de leur paysage.

Dans la réédition de L'Envers et l'Endroit, en I958, Camus écrit :

"Chaque artiste garde ainsi, au fond de lui, une source unique qui alimente pendant sa vie ce qu'il est et ce qu'il dit [...] Pour moi, je sais que ma source est dans L'Envers et l'endroit, dans ce monde de pauvreté et de lumière où j'ai longtemps vécu ».

Arrêtons-nous aussi à cette magnifique exergue de Médée qu'il citera au début de Retour à Tipasa (1954) : «Tu as navigué d'une âme furieuse loin de la demeure paternelle, franchissant les doubles rochers de la mer, et tu habites une terre étrangère ».

Dans son texte La mer au plus près, il décrit ainsi son exil et son paysage :

«J'ai grandi dans la mer et la pauvreté m’a été fabuleuse, puis j’ai perdu la mer, tous les luxes alors mont paru gris, la misère intolérable. Depuis, j'attends. J'attends les navires du retour, la maison des eaux, le jour limpide. Je patiente, je suis poli de toutes mes forces. On me voit passer dans les belles rues savantes, $\mathrm{j}$ 'admire les paysages, j'applaudis comme tout le monde, je donne la main. Ce n'est pas moi qui parle. ».

L'exil dibien est plus déchirant, c'est un cri pathétique qui souligne l'absence forcée de celui qu'on a chassé de son paradis. Il dit dans Les terrasses d'Orsol: « Qu'on me rende ma ville, que je puisse rencontrer des visages qui me parlent, des visages dont je puisse faire le tour, comme on fait chez nous pour le plaisir de la promenade le tour des remparts, comme on boit du thé à l'ombre des platanes, comme on court au devant de la mer, affronte de la poitrine cette mer miterreine nôtre, vacillant sous le point du soleil » (pp.85-86). Il aura le lyrisme du poète et la précision du topographe qui va de la Médresse à la maison du dhikr en passant par les Jardins de l'Éternité.

Ce parcours initiatique qui ne cessera jamais est une exploration incessante de l'écrivain au sein d'un monde organique qu'il réinvente à chaque livre :

«Ceux qui ont eu la curiosité de me lire pourront en témoigner. De l'un à l'autre de mes livres, des passerelles sont jetées, non d'une manière calculée 
mais comme la conséquence naturelle d'une manière de procéder, traverses qui relient chaque livre à un autre, nullement dans une succession logique, mais organique. Car ce n'est pas une suite romanesque, ou poétique, que je me suis efforcé de mettre sur pied, j'ai été tenté au contraire par l'aventure que constitue une exploration tous azimuts » (L'Arbre à dires : 208).

Ce travail sur lui-même, cette exploration intérieure est toujours doublée du même procédé extérieur. Dans une interview intitulée «Mohammed Dib ou le regard intérieur " l'écrivain souligne l'importance pour lui de nommer en littérature les personnages et les lieux de ses livres :

"Avec La Grande Maison, avec L'Incendie, et Le Métier à tisser, le point de départ- j'ai dit que c'était un constat- c'était aussi un acte de foi. Il consistait en cela qu'il nommait ce qui n'avait pas encore été nommé, en littérature bien sûr. On n'avait pas encore vu dans les livres ces personnages qu'on trouve dans la trilogie. Ces personnages n'avaient pas d'existence littéraire. Les lieux non plus. Même s'il y avait eu précédemment des écrivains français ou algériens de souche française installés en Algérie, qui avaient écrit et situé des œuvres en Algérie, mais disons qu'il n'y avait pas cette intimité avec le paysage. Nos œuvres se sont trouvées, par la force des choses, davantage inscrites dans le paysage. Et dans ce paysage, même si certains de ses aspects ont déjà figuré dans des livres d'auteurs français, ce nétait pas dans le détail, dans l'intimité, dans la profondeur du paysage et surtout ce nétait pas dans la relation entre hommes, entre êtres humains et paysage. Or, c'est une relation fondamentale. C'est la relation qui est un des éléments de l'identité d'une personne ou d'un peuple. ».

Dans cette intervention, Dib utilise par deux fois le mot « intimité » mis en relation avec le paysage comme si l'intime ultime devait être lié à l'origine ou aux origines qui représentent le lien entre l'homme arabe et sa terre.

L'autre terre de l'écrivain c'est bien sûr sa langue. Le combat de Dib pour défendre son identité réelle avec une langue acquise à la force du poignet.

« [...] La langue française est à eux, elle leur appartient. Qu'importe, nous en avons chipé notre part et ils ne pourront plus nous l'enlever. Ils n'attendent pas moins que nous les en remercions.

Mais qui, des uns, devraient remercier les autres? Et si, parce que nous en mangeons aussi de ce gâteau, nous lui apportions quelque chose de 
plus, lui donnions un autre goût ? Un goût qu'ils ne lui connaissent pas. [...] cela irrite, cela dérange tout de même certains français de nous voir écrire dans leur langue. La preuve? A chacune de mes rencontres avec le public, il s'en trouve un qui me prend à partie et me somme de me justifier. Il m'est naturel d'écrire en français, que puis-je dire d'autre ? [...] Il faut se rendre à l'évidence. Nous ferons toujours partie de ces immigrés ou de ces bohémiens qui campent aux abords d'une ville et sont soupçonnés de voler les poules de l'autochtone $[. .$.$] je reste donc là à camper sur le$ terrain vague d'une langue».

L'amertume ironique de ce propos est une pierre sur le terrain vague dibien qui n'a jamais cessé de blessé mais qui ne l'a pas empêché de créer des liens avec des intellectuels d'Afrique du Nord. Pourtant, après de grandes tentatives de partage et la création de revues littéraires souvent éphémère comme Forge, Soleil, puis Terrasses et Simoun l'inévitable rupture aura lieu.

Dib traverse la période coloniale et sa décadence, la période de décolonisation et finalement l'indépendance, et à chaque pas, son œuvre est en dialogue intérieur/extérieur avec cette périphérie que représente la colonie par rapport à la métropole qui se veut et se dit le centre de toutes rencontres.

Les journées de Sidi Madani et les contacts avec Parain, Tortel, Guilloux, Cayrol (qui le mène aux éditions du Seuil) Camus, lui permettent, dans sa première étape, de commencer à publier ses romans et poèmes en France.

Il est le poète du bouleversant poème Sur la terre errante qui s'adresse à la terre algérienne, celui qui devra partir pour ne plus revenir en dépit des tentatives; mis à l'écart.

Le parcours de l'écrivain algérien Mohammed Dib se termine en 2003 dans l'accomplissement d'une œuvre dense, diverse et combattive.

Camus meurt accidentellement en I960 mais en 1994, la parution posthume de son roman autobiographique Le premier homme remet en scène l'écrivain à un moment où les violences se déchaînent dans son pays d'origine et conduisent à une réévaluation de l'Histoire nationale.

Assia Djebar dans son texte Ces voix qui m'assiègent (1999) revient sur Le premier homme et sur l'identité de Camus, auteur algérien : 
"Et je souris à celui qui, dans une des notes accompagnant le texte inachevé, remarquait à propos de son double : « Ce qu'ils n'aimaient pas en lui, c'était l'Algérien. » Justement, je souris à cet algérien -là [...] parce qu'écrivain, parce que femme et parce qu'Algérienne. »

Et S'adressant à ce Camus algérien, elle dira :

«Mon presque compatriote par la terre et l'espace d'enfance, plutôt que par l'histoire ».

Rendons hommage aux paysages, à la beauté des textes camusiens, ceux de L'Été et de Noces.

Paysages naturels de Tipasa et Djemila, paysages urbains de la ville d'Oran, dans le Petit Guide pour des villes sans passé.

Dib et Camus laissent en héritage dans leurs œuvres un regard et une écriture des paysages de l'Algérie qui fait d'eux ce que Camus appelaient des « frères de soleil ».

A titre de conclusion, rappelons les paroles de Jean Grenier dans son prologue aux «Inspirations Méditerranéennes » publiées en I939. Il y disait :

«Il existe pour chaque homme des lieux prédestinés au bonheur, des paysages où il peut s'épanouir er connaître, au-delà du simple plaisir de vivre, une joie qui ressemble à un ravissement, une de ces joies dont parle Flaubert : "J'ai entrevu quelquefois un état de l'Âme supérieur à la vie, pour qui la gloire ne serait rien, et le bonheur même, inutile ».

La Méditerranée peut inspirer un tel état de l'âme. Elle ne risque pas de jeter dans cette confusion de sentiments qui faisait voir aux Romantiques dans les paysages un aliment spirituel ou même une intuition du divin. Par les lignes et les formes qu'elle impose elle rend la vérité inséparable du bonheur ; l'ivresse même de la lumière n'y fait qu'exalter l'esprit de contemplation. » 


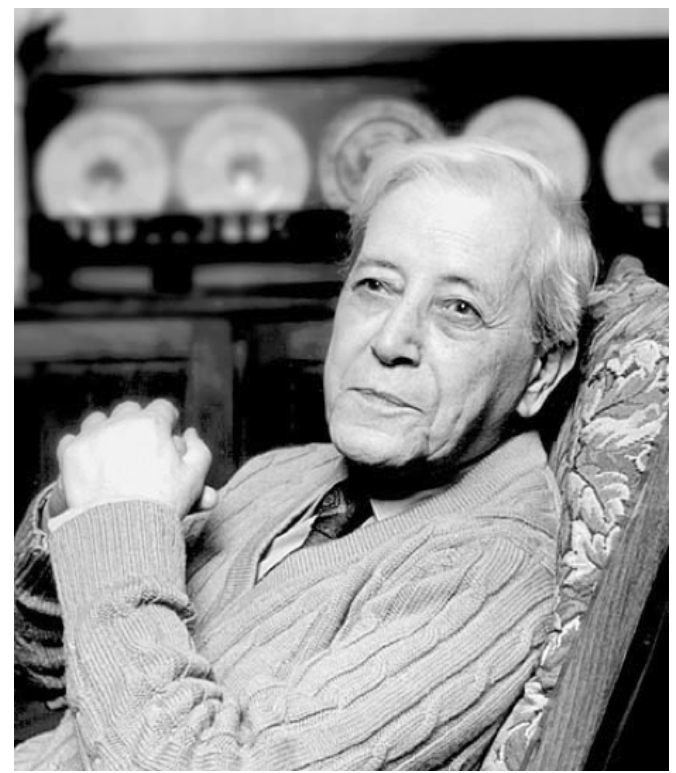

Mohamed Dib

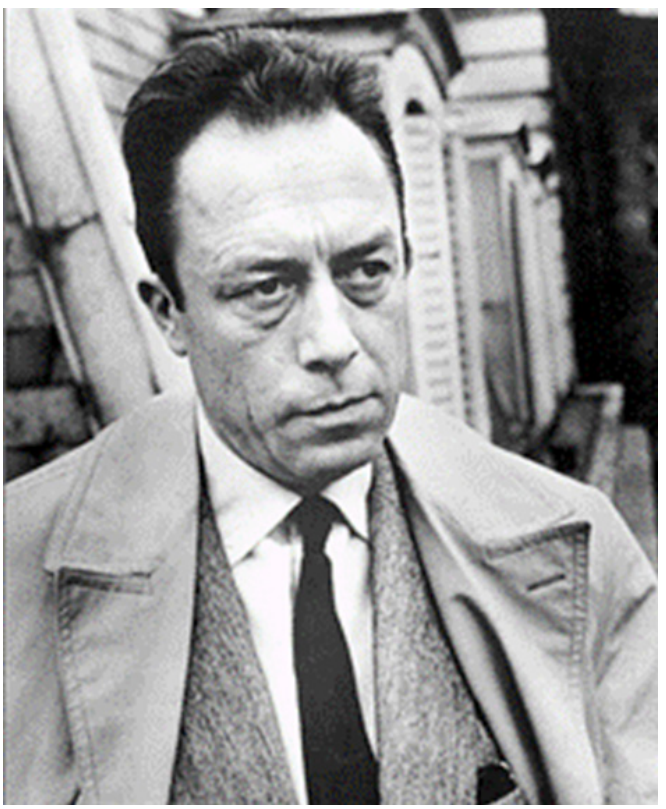

Albert Camus 


\section{BIBLIOGRAPHIE}

CAmus, Albert. L'Été, Paris, Gallimard, I954.

Camus, Albert. Le Premier Homme, Paris, Gallimard, 1994.

Camus, Albert. Noces, Paris, Gallimard, I950.

Camus, Albert. L'Envers et l'Endroit, Paris, Gallimard, 1958.

Dıв, Mohammed. L'Arbre à dires, Essai, Paris, Albin Michel, I998.

Dів, Mohammed. L'Incendie, Paris, Le Seuil, I954.

Diв, Mohammed. La grande maison, Paris, Le Seuil, 1952.

Dıв, Mohammed. Le Métier à tisser, Paris, Le Seuil, I957.

Dів, Mohammed. Les terrasses d'Orsol, Paris, Sindbad, 1985.

Diв, Mohammed. Poésie, Euvres Complètes, La Différence, 2007.

Diв, Mohammed, Tlemcen ou les lieux de l'écriture, textes et photos de philippe Bordas, Paris, Bordas, La Revue Noire, 1994.

Djebar, Assia. Ces voix qui miassiègent, Paris, Albin Michel, 1999.

Grenier, Jean. Inspirations Méditerranéennes, Paris, Gallimard, 1939. 